\title{
Nilpotent Singer groups
}

\author{
Nick Gill* \\ 9 Leonard Road, Redfield, Bristol, BS5 9NS, UK. \\ nickgill@cantab.net
}

Submitted: Jun 11, 2006; Accepted: Oct 10, 2006; Published: Oct 27, 2006

Mathematics Subject Classification: 20B25, 51A35

\begin{abstract}
Let $N$ be a nilpotent group normal in a group $G$. Suppose that $G$ acts transitively upon the points of a finite non-Desarguesian projective plane $\mathcal{P}$. We prove that, if $\mathcal{P}$ has square order, then $N$ must act semi-regularly on $\mathcal{P}$.

In addition we prove that if a finite non-Desarguesian projective plane $\mathcal{P}$ admits more than one nilpotent group which is regular on the points of $\mathcal{P}$ then $\mathcal{P}$ has non-square order and the automorphism group of $\mathcal{P}$ has odd order.
\end{abstract}

\section{Introduction}

A Singer group $S$ of a projective plane $\mathcal{P}$ of order $x$ is a collineation group of $\mathcal{P}$ which acts sharply transitively on the points of $\mathcal{P}$. The existence of such a Singer group is equivalent to a $(v, k, 1)$ difference set in $S$ where $v=x^{2}+x+1, k=x+1$ and the associated $2-(v, k, 1)$ design is isomorphic to $\mathcal{P}$.

Ho [Ho98, theorem 1] has proved the following theorem concerning abelian Singer groups:

Theorem C. A finite projective plane which admits more than one abelian Singer group is Desarguesian.

We will present an alternative proof of this theorem (our proof, unlike Ho's, will be dependent on the Classification of Finite Simple Groups) and then will present work aimed at extending the result to nilpotent Singer groups. In particular we prove the following:

Theorem B. Suppose that a non-Desarguesian finite projective plane $\mathcal{P}$ of order $x$ admits more than one nilpotent Singer group. Then the automorphism group of $\mathcal{P}$ has odd order and $x$ is not a square.

*I wish to thank the University of Western Australia and the University of Gent for their support during the writing of this paper. 
In the course of proving Theorem B we will need to prove the following:

Theorem A. Let $F$ be a nilpotent group which is normal in a transitive automorphism group $G$ of $\mathcal{P}$, a projective plane of order $x=u^{2}$. Then $F$ acts semi-regularly on $\mathcal{P}$.

\section{Background results}

Let $\mathcal{P}$ be a projective plane of order $x$ and suppose that $\mathcal{P}$ admits $G$ a point-transitive automorphism group. It is well known that an involution $g \in G$ must fix $x+1, x+2$ or $x+\sqrt{x}+1$ points of $\mathcal{P}$; furthermore no automorphism of $\mathcal{P}$ may fix more than $x+\sqrt{x}+1$ points of $\mathcal{P}$ ([Dem97, 4.1.7]). If either of the first two possibilities hold then $g$ is known as a perspectivity; Wagner has proved that if $\mathcal{P}$ admits a perspectivity then $\mathcal{P}$ is Desarguesian and $P S L(3, x) \unlhd G \leq P \Gamma L(3, x)$ ([Wag59]).

In order to prove Theorems $\mathrm{A}, \mathrm{B}$ and $\mathrm{C}$, it is not necessary to consider the situation where $\mathcal{P}$ is Desarguesian and $P S L(3, x) \unlhd G \leq P \Gamma L(3, x)$. Hence for the remainder of this paper we will operate under the following hypothesis.

Hypothesis. Let $\mathcal{P}$ be a projective plane of order $x>4$. Let $G$ be an automorphism group of $\mathcal{P}$ which acts transitively upon the points of $\mathcal{P}$. If $G$ contains any involutions then $x=u^{2}, u>2$, and each involution fixes $u^{2}+u+1$ points. If $h \in G$ then $h$ fixes at most $x+\sqrt{x}+1$ points.

We require the following result which is dependent on the Classification of Finite Simple Groups:

Theorem 1. [Gil, Theorem A] Suppose that G acts transitively on the points of a projective plane $\mathcal{P}$ of order $x$. Then one of the following cases holds:

- $\mathcal{P}$ is Desarguesian, $P S L(3, x) \unlhd G \leq P \Gamma L(3, x)$ and the action is 2-transitive on points;

- the Fitting group and the generalized Fitting group of $G$ coincide, i.e. $F^{*}(G)=$ $F(G)$.

Clearly, under our hypothesis, Theorem 1 implies that the Fitting group and the generalized Fitting group of $G$ coincide, i.e. $F^{*}(G)=F(G)$.

We will also need the following result of Ljunggren:

Lemma 2. [Lju43, p.11] If $u^{2}+u+1=p_{1}^{a}$ where $p_{1}$ is a prime, then either $p_{1}^{a}=p_{1}$ or $p_{1}^{a}=7^{3}$.

Write $\alpha$ for a point of $\mathcal{P}$. For a collineation group $H$ of $\mathcal{P}$, write $H_{\alpha}$ for the stabilizer of $\alpha$. 


\section{$3 \quad$ Nilpotent collineation groups}

In order to prove Theorem A we need a well known result of Camina and Praeger. We state a weaker version which is sufficient for our purposes:

Theorem 3. [CP93, Theorem 1] Let $G$ act transitively on the points of a projective plane $\mathcal{P}$. Let $N$ be a normal subgroup of $G$. Then $N$ acts faithfully on each of its point orbits.

Note that, in particular, Theorem 3 implies that a minimal normal subgroup of $G$ will act semi-regularly on the points of $\mathcal{P}$. We are now in a position to prove Theorem A.

Proof. Let $N$ be a Sylow $p$-group of $F$ for some prime $p$ dividing the order of $F$. Suppose that $N$ does not act semi-regularly. If $\left|N: N_{\alpha}\right|=p$, a prime, then, since $N$ acts faithfully, $N<S_{p}$, the Symmetric group on $p$ letters. But then $|N|=p$ and $N$ is semi-regular which is a contradiction. Thus $\left|N: N_{\alpha}\right| \geq p^{2}$ and $x>25$.

Observe that the average number of fixed points for non-identity elements of $N$ is

$$
\left(x^{2}+x+1\right) \frac{\left|N_{\alpha}\right|-1}{|N|-1}>\frac{x^{2}+x+1}{2\left|N: N_{\alpha}\right|} .
$$

Now $\left|N: N_{\alpha}\right|$ divides into $u^{2} \pm u+1$. If $\left|N: N_{\alpha}\right|<u^{2} \pm u+1$ then a non-identity element of $N$ fixes, on average, more than $\frac{3}{2}\left(u^{2}-u+1\right)>u^{2}+u+1$ fixed points which contradicts our hypothesis.

If $\left|N: N_{\alpha}\right|=u^{2} \pm u+1$ then, by Lemma $2,\left|N: N_{\alpha}\right|=7^{3}$.

If $\left|N: N_{\alpha}\right|=7^{3}=u^{2}-u+1$ and $\left|N_{\alpha}\right|>7$ then the average number of fixed points for non-identity elements of $N$ is

$$
\left(x^{2}+x+1\right) \frac{\left|N_{\alpha}\right|-1}{|N|-1}=343 \times 381 \frac{\left|N_{\alpha}\right|-1}{7^{3}\left|N_{\alpha}\right|-1}>\frac{343 \times 381}{351}>370 .
$$

Now $x=361$ and an element of order 7 must fix a multiple of 7 points. This implies that the most number of points such an element may fix is 357 which is a contradiction.

If $\left|N: N_{\alpha}\right|=7^{3}=u^{2}-u+1$ and $\left|N_{\alpha}\right|=7$ then $|A u t N|$ is not divisible by 127 . Thus $F(G)$ must contain a non-trivial Sylow 127-group and $G$ has a normal semi-regular subgroup $P$ of order 127 . Furthermore $P$ centralizes $N_{\alpha}$ and so

$$
\left|F i x\left(N_{\alpha}\right)\right| \geq 7 \times 127>x+\sqrt{x}+1 .
$$

This is a contradiction.

If $\left|N: N_{\alpha}\right|=7^{3}=u^{2}+u+1$ then $x=324$. In fact $N=F=F(G)$ since otherwise $F(G)>N$ and a point semi-regular group of order 307 must centralize $N$ which is impossible.

By Theorem 3 the centre of $N$ acts semi-regularly on the points of $\mathcal{P}$. This implies that $N_{\alpha}$ fixes a subplane. Suppose that $|Z(N)|>7$; then $N_{\alpha}$ must fix a subplane of order 18. Thus, for $g \in N_{\alpha}, F i x(g)=F i x\left(N_{\alpha}\right)$. Then take $\beta$ a point of $\mathcal{P}$ not in $\operatorname{Fix}\left(N_{\alpha}\right)$. We must have $N_{\alpha} \cap N_{\beta}=\{1\}$. Thus $\left|N_{\beta}\right| \leq 7^{3}$ and so $|N| \leq 7^{6}$. But in this case 307 does not divide into $\mid$ Aut $N \mid$ which is a contradiction. 
Suppose, alternatively, that $|Z(N)|=7$. Let $X=N_{N}\left(N_{\alpha}\right)$. If $X>N_{\alpha} Z(N)$ then there are at most $7 N$-conjugates of $N_{\alpha}$ and so $N_{\alpha}$ must fix 0 or a multiple of 49 points in each orbit of $N$. Hence $N_{\alpha}$ fixes a Baer subplane and our previous argument can be applied.

Thus we assume that $X=N_{\alpha} Z(N)$. Since $N$ is nilpotent $N_{N}(X)>X$ and so we choose $\alpha$ and $\beta$ points in the same $N$-orbit such that $N_{\alpha}$ and $N_{\beta}$ are distinct subgroups of $N_{\alpha} Z(N)$. Then $\left|N_{\alpha}:\left(N_{\alpha} \cap N_{\beta}\right)\right|=7$. Furthermore $Y:=N_{N}\left(N_{\alpha} \cap N_{\beta}\right) \geq\langle X, n\rangle>X$, where $n \in N$ such that $\alpha n=\beta$, hence $Y$ acts on the fixed set of $N_{\alpha} \cap N_{\beta}$ with orbits of size a multiple of 49. We conclude that $N_{\alpha} \cap N_{\beta}$ fixes a Baer subplane.

If $N_{\alpha} \cap N_{\beta}$ fixes an entire $N$-orbit then, since $N$ acts faithfully on its point-orbits, $N_{\alpha} \cap N_{\beta}=\{1\}$. Thus $|N|=7^{4}$. Alternatively $N_{\alpha} \cap N_{\beta}$ fixes exactly 0 or 49 points in any $N$-orbit. In which case we can find two other points $\gamma$ and $\delta$ in the same $N$-orbit as $\alpha$ such that $N_{\gamma} \cap N_{\delta}$ fix a Baer subplane. Then $\left(N_{\alpha} \cap N_{\beta}\right) \cap\left(N_{\gamma} \cap N_{\delta}\right)=\{1\}$ and so $|N| \leq 7^{8}$. Thus in all cases $|N| \leq q^{8}$ and 307 does not divide into $\mid$ Aut $N \mid$ which is a contradiction.

\section{Abelian Singer groups}

Throughout this section $\mathcal{P}$ is non-Desarguesian and $S \leq G$ is an abelian Singer group of $\mathcal{P}$.

We record some results of Ho [Ho98]:

Theorem 4. [Ho98, theorem 2] An abelian Singer group contained in a soluble collineation group of a finite projective plane is always normal.

Lemma 5. [Ho98, lemma 4.3] Let $Q<G$ be a collineation group normalized by $S$. Then $S$ centralizes $Q$ if one of the following holds:

1. $Q$ is abelian;

2. $|Q|$ is prime to $|S|$;

3. $x=u^{2}$ and $Q$ is nilpotent.

We are now able to give an alternative proof to Theorem C.

Proof. Suppose that $S$ and $T$ are abelian Singer groups lying in $G$. We need to prove that $S=T$.

If $G$ is soluble then both $S$ and $T$ are normal in $G$ and so lie in $F(G)$. By Lemma 5 , $S$ and $T$ centralize each other. Thus $\langle S, T\rangle$ is abelian and transitive on the points of $\mathcal{P}$. By Theorem 3, $|\langle S, T\rangle| \leq v$ and so $S=T$.

If $G$ is not soluble then we may assume that $G$ contains a Baer involution. Hence $x=u^{2}$ and, by Lemma $5, S$ and $T$ both centralize $F(G)$. Since $F(G)=F^{*}(G)$ this means that $F(G)$ contains both $S$ and $T$. Now $F(G)$ is soluble and so we can apply the same argument as when $G$ was soluble and conclude that $S=T$. 


\section{$5 \quad$ Nilpotent Singer groups}

Throughout this section $\mathcal{P}$ is non-Desarguesian and $S \leq G$ is a nilpotent Singer group of $\mathcal{P}$.

Lemma 6. $S$ contains $F(G)$.

Proof. Suppose the result does not hold and $S \nsupseteq F(G)$. Let $P \in S y l_{p} F(G)$ with $P \not \leq S$. Let $H:=P S=S P$.

Let $Q \in S y l_{p} H$ such that $Q=P P_{1}$ where $P_{1} \in S y l_{p} S$. Then consider $h^{-1} Q h$ for $h \in H$. We can write $h=s p$ where $p \in P$ and $s \in S$. Then

$$
h^{-1} Q h=p^{-1} s^{-1} P P_{1} s p=P p^{-1} s^{-1} P_{1} s p=P P_{1}^{p}=Q .
$$

Thus $Q$ is normal in $H$. But this is a nilpotent group normal in a transitive group hence, by Theorem A, $Q$ is semi-regular. But $|Q|$ does not divide into $x^{2}+x+1$ and we have a contradiction.

Corollary 7. Any prime dividing into $v$ divides into $|F(G)|$.

Proof. Since $C_{G}(F(G)) \leq F(G)$ we know that if $p$ divides into $|S|$ then $p$ divides into $|F(G)|$. So if $p$ divides into $v$ then $p$ divides into $|F(G)|$.

We are now in a position to prove Theorem B:

Proof. Assume, for the sake of contradiction, that $\mathcal{P}$ admits two distinct nilpotent Singer groups $S$ and $T$ and set $G:=A u t \mathcal{P}$. Let $v=p_{1}^{a_{1}} \ldots p_{r}^{a_{r}}$. Then $F(G)=N_{1} \times \cdots \times N_{r}$, $1 \neq N_{i} \in S y l_{p_{i}} F(G)$. In a similar way write,

$$
S=P_{1} \times \cdots \times P_{r}, \quad T=Q_{1} \times \cdots \times Q_{r} .
$$

We will assume, without loss of generality, that $P_{1} \neq Q_{1}$. Now $G \leq\left(N_{1} \times \cdots \times\right.$ $\left.N_{r}\right) \cdot\left(A_{1} \times \cdots \times A_{r}\right)$ where $A_{i}$ is a subgroup of the outer automorphism group of $N_{i}$. Then both $P_{i}$ and $Q_{i}$ lie in $\left(1 \times \cdots \times 1 \times N_{i} \times 1 \times \cdots \times 1\right) .\left(1 \times \cdots \times 1 \times A_{i} \times 1 \times \cdots \times 1\right)$. This implies that

$$
C_{G}\left(\left\langle P_{1}, Q_{1}\right\rangle\right) \geq P_{2} \times \cdots \times P_{r} .
$$

Now $\left\langle P_{1}, Q_{1}\right\rangle$ must contain an element $1 \neq g$ which fixes a point (since $P_{1} Q_{1}$ must have an orbit of size strictly less than $\left|P_{1} Q_{1}\right|$.) Then we know that

$$
\frac{|v|}{|v|_{p_{1}}}|| F i x g \mid \text {. }
$$

In fact, consider $\left\langle Q_{1}, P_{1}\right\rangle$ acting on $\mathcal{P} /\left(P_{2} \times \cdots \times P_{r}\right)$. Either this is a Frobenius action or there exists $g \in\left\langle Q_{1}, P_{1}\right\rangle$ that fixes more than one element. But a Frobenius action has a normal Frobenius kernel which must be $Q_{1}$ and $P_{1}$. This is a contradiction. Thus

$$
\frac{|v|}{|v|_{p_{1}}}<\mid \text { Fix } g \mid
$$

Now, since $x=u^{2}, v=(x+\sqrt{x}+1)(x-\sqrt{x}+1)$. The bracketed terms are coprime and so $\mid$ Fix $g \mid \geq 2(x-\sqrt{x}+1)>x+\sqrt{x}+1$. This gives a contradiction. 


\section{References}

[CP93] Alan R. Camina and Cheryl E. Praeger, Line-transitive automorphism groups of linear spaces, Bull. London Math. Soc. 25 (1993), 309-315.

[Dem97] P. Dembowski, Finite geometries, Springer-Verlag, 1997.

[Gil] Nicholas Gill, Transitive projective planes, Submitted.

[Ho98] Chat Yin Ho, Finite projective planes with abelian transitive collineation groups, J. Algebra 208 (1998), 533-550.

[Lju43] W. Ljunggren, Einige bemerkungen über die Darstellung ganzer Zahlen durch binäre kubische Formen mit positiver Diskriminante, Acta. Math. 74 (1943), $1-21$.

[Wag59] A. Wagner, On perspectivities of finite projective planes, Math. Z. 71 (1959), $113-123$. 